\title{
Manejo expectante de osteocondroma solitario interóseo de la tibia distal: reporte de un caso y revisión de la literatura
}

\section{Non-surgical Management of an Interosseous Solitary Osteochondroma of the Distal Tibia: A Case Report and Review of the Literature}

\author{
Clara Schulze S. ${ }^{1}$ Gonzalo Valenzuela G. ${ }^{1}$ Tomás Zamora H. ${ }^{1,2}$ \\ ${ }^{1}$ Escuela de Medicina, Pontificia Universidad Católica de Chile, \\ Santiago, Chile \\ ${ }^{2}$ Departamento de Ortopedia y Traumatología. Escuela de Medicina, \\ Pontificia Universidad Católica de Chile, Santiago, Chile

\begin{abstract}
Address for correspondence Dr. Tomás Zamora Helo, MD, Departamento de Ortopedia y Traumatología, Escuela de Medicina, Pontificia Universidad Católica de Chile, Diagonal Paraguay 362, Santiago, Chile 8330077 (e-mail: tzamora@med.puc.cl).
\end{abstract}

\begin{abstract}
Resumen
Palabras clave

- osteocondroma

- tibia distal

- manejo expectante

- reporte de caso

La localización del osteocondroma en la tibia distal es poco frecuente y su crecimiento con compromiso y deformidad del peroné distal es aún más raro, haciéndolo una condición más sintomática que en otras ubicaciones. Factores como la severidad de los síntomas, deformidad progresiva del tobillo, complicaciones sindesmóticas, riesgo de fractura patológica o transformación maligna, junto con nuevas y mejores técnicas quirúrgicas, han llevado a que el manejo expectante de esas lesiones sea excepcional y escasamente reportado. Presentamos el caso de un joven de 17 años con osteocondroma solitario interóseo tibio distal sintomático y compromiso fibular, que fue exitosamente manejado en forma expectante. A los 5 años de seguimiento clínico-radiológico no presenta complicaciones y la lesión se mantiene estable.

Osteochondromas located in the distal tibia are a rare condition, and the involvement of the distal fibula with deformity is even more uncommon. Factors such as the severity of symptoms, progressive deformity of the ankle, syndesmotic complications, the risk of pathological fracture or malignant transformation, together with new and safer surgical techniques, have led to scarce reports of non-surgical management. We present a case report of a 17-year-old male with a symptomatic interosseous solitary osteochondroma in the distal tibia with fibular involvement, which was successfully managed non surgically. After 5 years of clinical and radiological follow-up, he has no complications, and the lesion remains stable.
\end{abstract}

received

September 22, 2018 accepted

December 12, 2018

published online

February 21, 2019
DOI https://doi.org/

10.1055/s-0039-1677832. ISSN 0716-4548.
Copyright (e 2018 by Thieme Revinter

Publicações Ltda, Rio de Janeiro, Brazil
License terms

c) $(1) \$$ 


\section{Introducción}

Los osteocondromas son las lesiones tumorales benignas más frecuentes. Corresponden a exostosis cartilaginosas preferentemente solitarias, ${ }^{1}$ pero que también pueden encontrarse en el contexto de una exostosis múltiple.

Su localización en tibia y peroné distal son poco frecuentes, reportándose entre el 1\% y el12\% según algunas series. Suele presentarse clínicamente como una deformidad en el tobillo que aumenta en la etapa de crecimiento, a lo cual se puede asociar dolor, compresión de nervios periféricos o reducción de la movilidad articular. ${ }^{2,3}$ Por otro lado, en el seguimiento se describen deformidades que pueden afectar la estabilidad de la mortaja tibio astragalina, alteraciones de la sindesmosis, acortamientos y deformidades angulares que condicionan un tobillo en valgo o varo y finalmente pueden producir cambios degenerativos. ${ }^{4,5}$ Las fracturas a nivel del tallo del pedículo del tumor son raras, ${ }^{6}$ aunque puede presentarse en ese tipo de lesiones. El riesgo de malignización es bajo, pero debe descartarse ante aumento del dolor, crecimiento o alteración de criterios imagenológicos. ${ }^{7}$

En los últimos años se ha propuesto el tratamiento quirúrgico como una alternativa válida en pacientes sintomáticos, especialmente en exostosis hereditarias múltiples y deformidades en valgo, sin embargo, su resección puede estar asociada a sinostosis tibiofibular, lesiones neurovasculares o inestabilidad del tobillo según el abordaje a utilizar. ${ }^{8,9}$ En este caso, presentamos la evolución natural a 5 años de un paciente con osteocondroma tibial distal con deformidad peroneal secundaria, sin complicaciones.

Se obtuvo el consentimiento informado por escrito del paciente para la publicación de este informe de caso y las imágenes que lo acompañan.

\section{Caso Clínico}

Paciente del sexo masculino de 17 años de edad, sin antecedentes médicos ni quirúrgicos relevantes, consulta por cuadro de un mes de evolución de dolor retromaleolar derecho sin irradiación ni síntomas neuropáticos que aparece en forma diaria en relación a la práctica de fútbol. Asociado a dificultad en la marcha y restricción leve a la eversión del pie. Además, refiere apreciar aumento de volumen a nivel del maléolo lateral de esa extremidad, sin historia de trauma local.

Al momento de la consulta el paciente presenta un estadio de Tanner 5, con talla $1,69 \mathrm{~cm}$, IMC $24.5 \mathrm{~kg} / \mathrm{m} 2$ y talla objetivo genética de $1,67 \mathrm{~cm}$. Al examen físico destaca aumento de volumen de consistencia firme de $4 \mathrm{~cm} \times 3 \mathrm{~cm}$ sobre el aspecto lateral del tobillo (- Fig. 1), liso, con márgenes definidos y no sensible a la palpación. Impresiona deformación hacia la porción superior del maléolo lateral derecho. Fuerza y sensibilidad de la extremidad conservadas, sin déficit neurovascular distal al examen. Su American Orthopaedic Foot $\mathcal{E}$ Ankle Society Score (AOFAS) es de 65 puntos (20 puntos en dolor, 35 puntos en función y 10 puntos en alineamiento). La flexión plantar y dorsal del tobillo es normal y simétrica con el lado contrario, mientras que el rango de movimiento del retropie (inversión-eversión), presenta una restricción leve (75-100\% comparado con el contralateral).

Las radiografías antero-posterior (AP), lateral y oblicua del tobillo ( - Fig. 2) mostraron una exostosis en la metáfisis lateral distal de la tibia, con continuidad cortical y trabecular con el hueso, de $25 \times 24 \mathrm{~mm}$, compatibles con osteocondroma. Destaca remodelación del peroné distal, con abombamiento y esclerosis reactiva de su cortical lateral. Espacio articular tibio-peroneo-astragalino de amplitud conservada.

En la Resonancia Magnética (RM) con contraste (-Fig. 3) destaca una lesión de $30 \times 25 \times 22 \mathrm{~mm}$, que desplaza y deforma el peroné distal a nivel metafisiario, provocando un aumento del diámetro anteroposterior con disminución del diámetro transverso. El capuchón cartilaginoso mide $7 \mathrm{~mm}$ y presenta discreta intensificación de señal post-gadolineo.

Se discuten con el paciente la historia natural y progresión de la patología, junto con los riesgos y beneficios de la escisión quirúrgica. Finalmente, se opta por diferir el manejo quirúrgico; indicándose evitar actividades de alto impacto y seguimiento clínico-radiológico periódico.

A los 5 años de seguimiento, presenta dolor intermitente controlado, sin inestabilidad ni limitaciones en las actividades de la vida diaria. Al examen físico no se aprecia crecimiento evidente y persiste sin compromiso neurovascular. Su puntaje

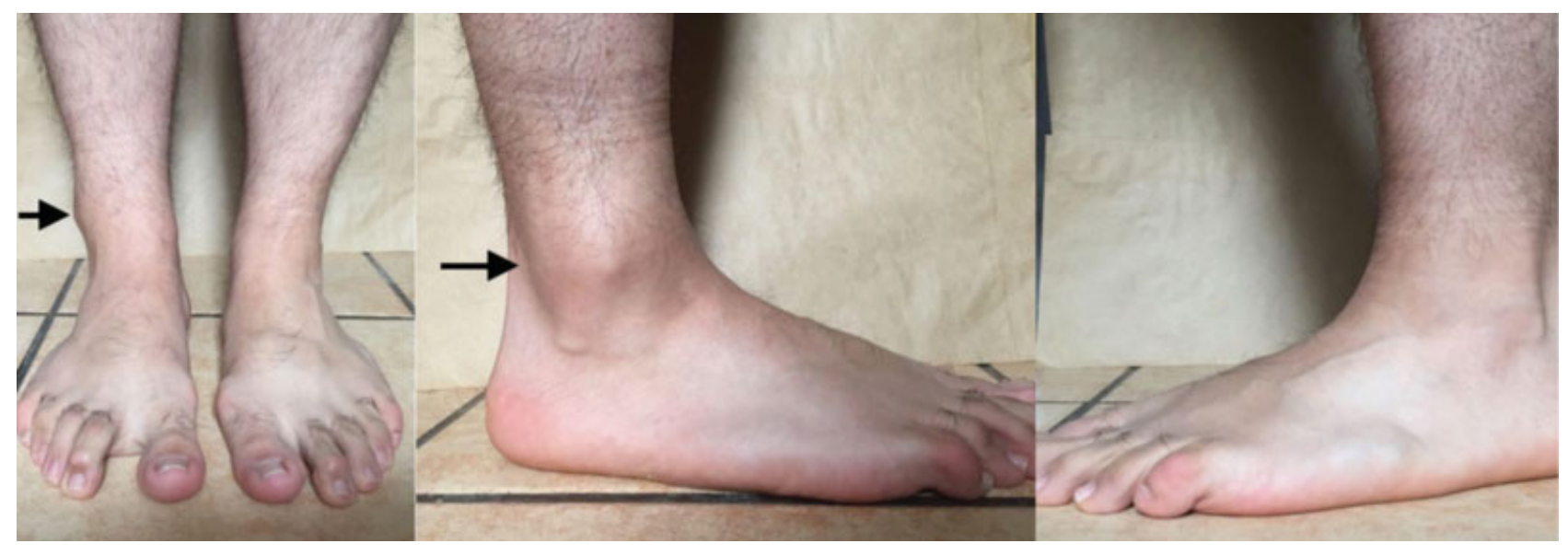

Fig. 1 Fotografía clínica del paciente que muestra lesión sobre el aspecto lateral del tobillo derecho. 


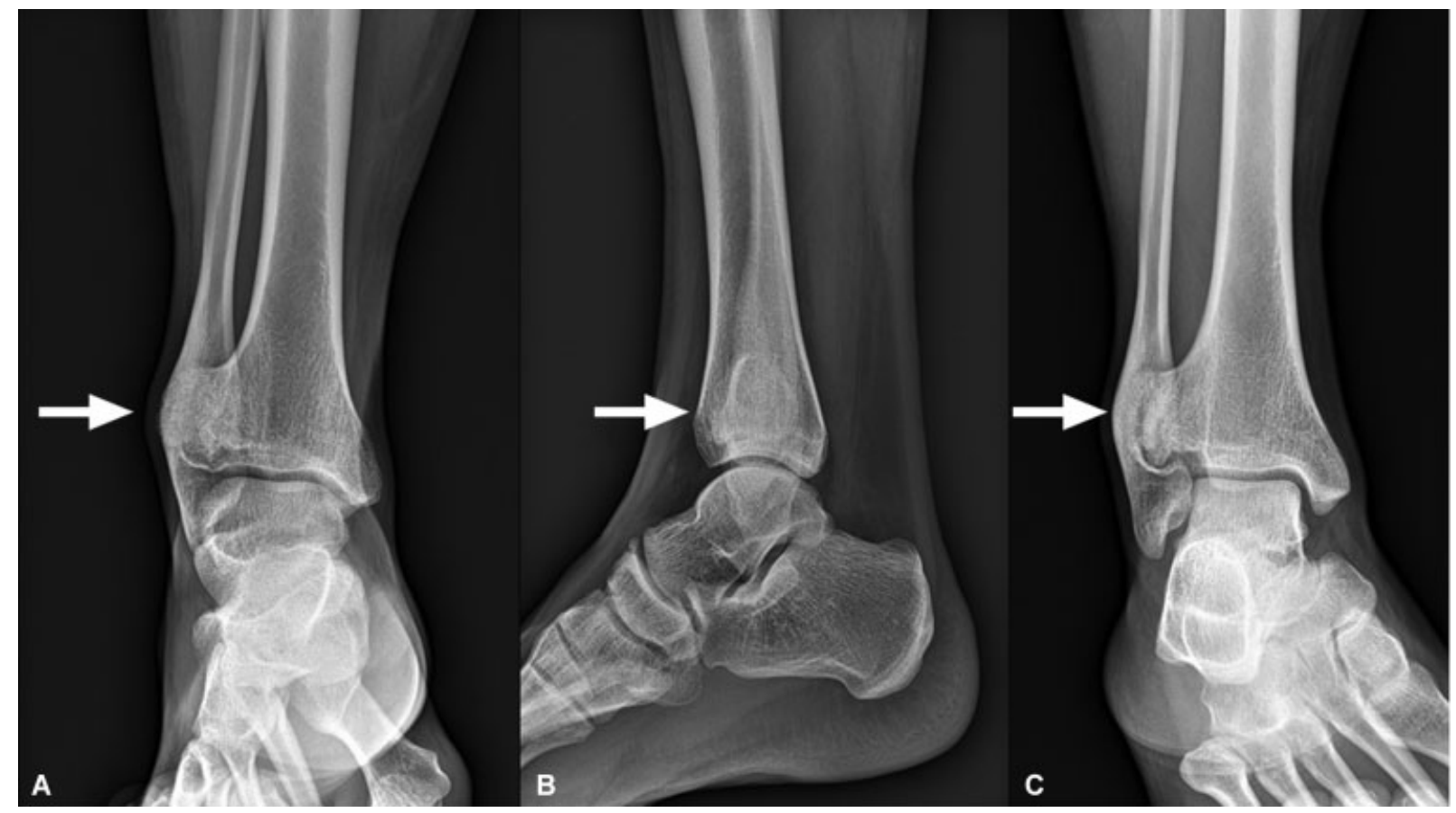

Fig. 2 Radiografía anteroposterior (A), lateral (B) y oblicua (C) del tobillo derecho al diagnóstico, mostrando exostosis ósea tibio distal lateral.

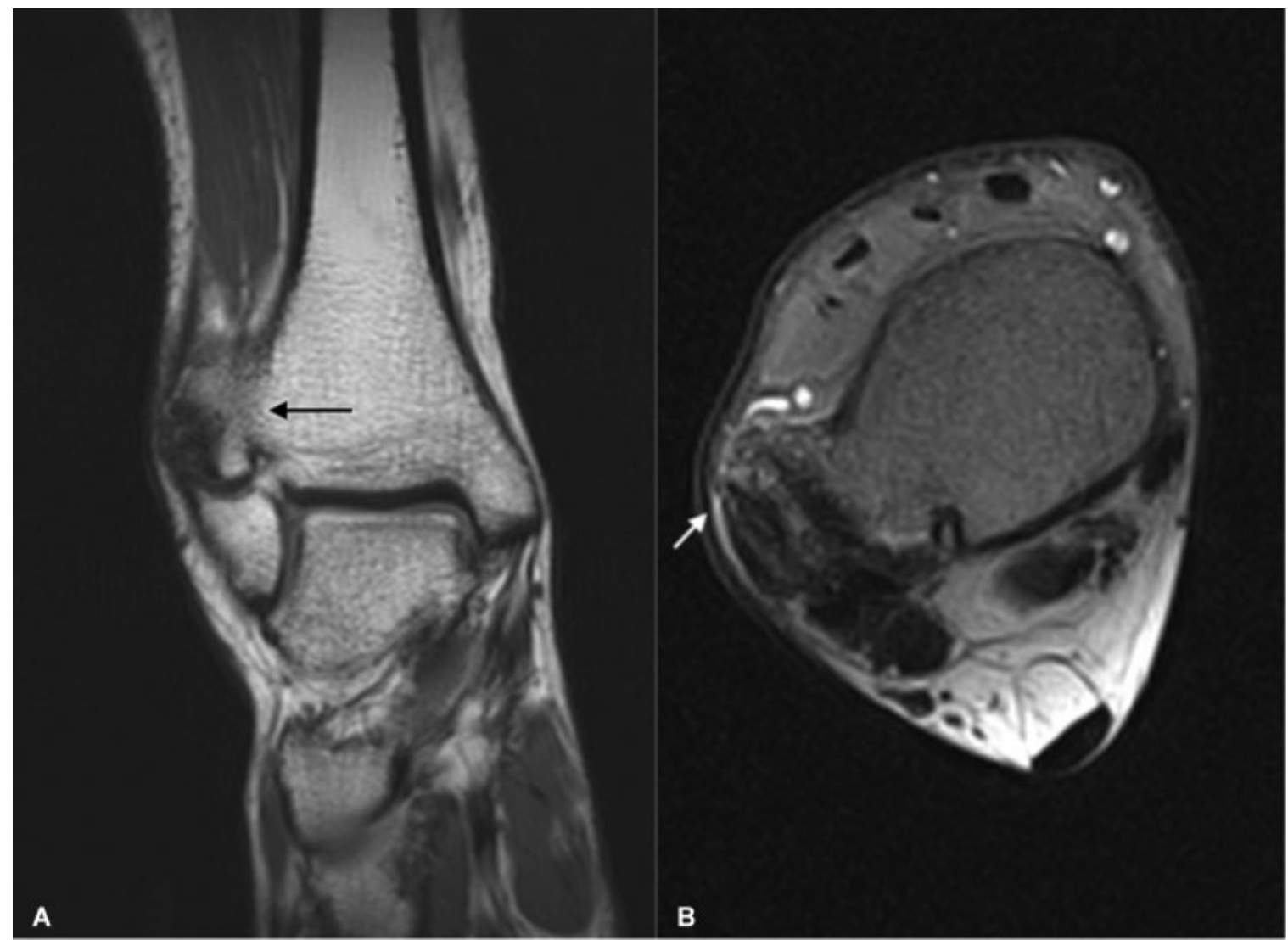

Fig. 3 Resonancia magnética de tobillo derecho. (A) Secuencia T1 coronal evidenciando desplazamiento y deformidad del peroné distal debido al osteocondroma. (B) Secuencia T1 saturación grasa con gadolinio mostrando capuchón cartilaginoso con discreta intensificación de señal. 


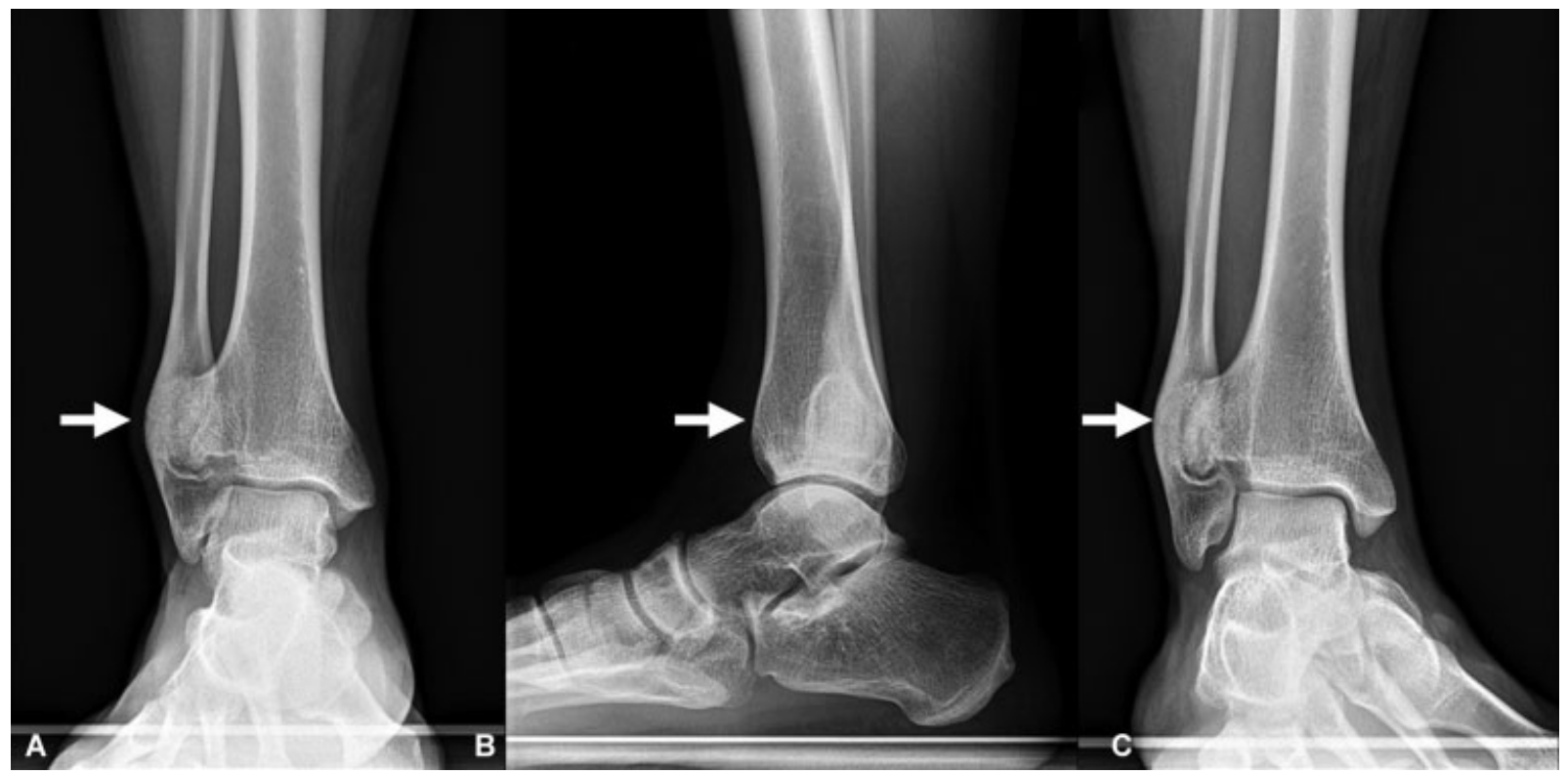

Fig. 4 Radiografía anteroposterior (A), lateral (B) y oblicua (C) del tobillo derecho, mostrando exostosis ósea tibio distal lateral al seguimiento a 5 años.

en el AOFAS score es de 85 puntos (30 puntos en dolor, 45 puntos en función y 10 punto en alineamiento) (66).

Las radiografías de control (-Fig. 4) no evidencian cambios significativos respecto al estudio anterior.

\section{Discusión}

El osteocondroma es el tumor óseo benigno biopsiado más común. ${ }^{4,10}$ Suele ubicarse en la metáfisis de huesos largos de crecimiento rápido durante la segunda década de la vida., ${ }^{41}$ Se localiza con frecuencia en las metáfisis alrededor de la rodilla, seguido por el húmero proximal y escápula. La afectación del extremo distal de la tibia representa menos del $4 \%$ de los casos y su origen desde el borde interóseo de la tibia distal con compromiso del peroné distal, es aún menos frecuente. ${ }^{12}$ Al contrario que en otras localizaciones, el osteocondroma de la tibia distal es a menudo sintomático, siendo su forma de presentación más frecuente el aumento de volumen y el dolor en el tobillo. ${ }^{4}$

Una deformación plástica a nivel de la epífisis tibial y del peroné distal, ocurre en pacientes sin madurez ósea, condicionando una deformación angular progresiva del tobillo. Si el tumor crece desde su origen en el borde interóseo de la tibia distal, puede deformar la tibia y el peroné hasta llevar a la fusión de la fisis. ${ }^{11}$ Otras complicaciones descritas comprenden cambios sindesmóticos, limitación en la función por deformidad o dolor y cambios degenerativos del tobillo, lo que muchas veces hace que el tratamiento quirúrgico sea de elección.

Nuestro paciente presentó un osteocondroma de tibia distal con el aumento de volumen y dolor como principal síntoma. Al presentarse posterior a su madurez esquelética, el riesgo de deformidad angular progresiva disminuye por lo que el manejo expectante sin las potenciales complicaciones de la cirugía debió ser ponderado con el riesgo de fractura, transformación maligna y persistencia o empeoramiento de las molestias.
Síntomas como dolor, aumento de volumen local, malestar en relación al ejercicio o limitación funcional, pueden sugerir tanto inflamación de la bursa como fractura, compresión de nervio periférico, pinzamiento del tibial posterior o incluso una transformación maligna. ${ }^{13-16}$ Es así como el estudio imagenológico es fundamental para poder descartar esas complicaciones. La radiografía simple en primera instancia es el estudio de elección para comenzar la investigación como se realizó en nuestro caso. Ante dudas diagnósticas, la tomografía computada (mejor visualización ósea) y la RM (mejor visualización de tejidos blandos) nos permiten además evaluar el riesgo de malignización y el espesor del capuchón cartilaginoso con sensibilidad y especificidad entre $95 \%-100 \%{ }^{7}$ Con respecto al uso de contraste endovenoso, y su evaluación dinámica, él permite evaluar mejor la vascularización tumoral y sus márgenes, siendo eso de mayor importancia en caso de tumores malignos y su planificación quirúrgica.

En nuestro caso, el estudio imagenológico permitió descartar la gran mayoría de esas complicaciones. Por otra parte, el seguimiento sin cambios por más de 5 años establece el diagnóstico según la gran mayoría de las recomendaciones sin necesidad de mayor estudio histológico.

Con respecto al tratamiento quirúrgico, las principales alternativas son el uso de un abordaje anterior clásico y un abordaje transfibular. En una serie de 8 pacientes con osteocondroma inter-tibiofibular distal sometidos a abordaje transfibular, a 6 años se objetivó una media de 92,4 puntos en el AOFAS score, resultados similares a los obtenidos en nuestro caso. Dentro de los riesgos de ese abordaje se encuentran la lesión de las estructuras neurovasculares posterolaterales, la sinostosis tibiofibular, inestabilidad del tobillo y la necesidad de nuevas intervenciones para retirar el material de osteosíntesis del peroné ${ }^{1,8}$. Por otro lado, el abordaje anterior tiene la desventaja de mayor dificultad técnica en 
lesiones posterolaterales y el daño de del paquete neurovascular anterior ${ }^{8}$.

Finalmente, se debe considerar el riesgo de transformación maligna. A partir de series quirúrgicas, el riesgo se ha estimado en $1 \%-2 \%,{ }^{13}$ con mayor riesgo en caso de pacientes con exostosis múltiple hereditaria. Respecto a signos radiológicos de malignidad, un capuchón de cartílago con grosor mayor a $2 \mathrm{~cm}$ ha demostrado elevada sensibilidad y especificidad para detectar esta complicacion. ${ }^{7}$ En nuestro caso, la RM mostró un capuchón de $7 \mathrm{~mm}$, apoyando características de benignidad.

Como conclusión, el manejo de un osteocondroma de tibia distal de forma expectante es algo factible y seguro en casos seleccionados. En su evolución natural, una vez alcanzada la madurez esquelética, el crecimiento del osteocondroma disminuye y finalmente cesa en casi todos los casos. A pesar de que la resolución espontánea está descrita en algunas series, eso es extremadamente infrecuente. ${ }^{17}$ Una vez descartadas las principales complicaciones de esas lesiones, es necesario valorar la sintomatología del paciente contra los potenciales riesgos descritos de la cirugía y en casos de duda, la observación por un periodo acotado puede otorgar los elementos clínicos necesarios para tomar la decisión. En el caso de presentarse con riesgo importante de deformidad o fractura, bloqueo mecánico, alteraciones importantes de la sindesmosis, compresión de estructuras neurovasculares, empeoramiento de los síntomas o signos sugerentes de malignización, el tratamiento quirúrgico está indicado. Si se opta por el tratamiento quirúrgico, una excisión completa mediante un abordaje anterior o transfibular son las alternativas más comúnmente utilizadas.

Fuente de Financiación

Este estudio no recibió financiamiento.

Declaración de Conflictos de Interés

Ninguno de los autores de este estudio presenta conflictos de interés.

\section{Bibliografía}

1 Thakur GB, Jain M, Bihari AJ, Sriramka B. Transfibular excision of distal tibial interosseous osteochondroma with reconstruction of fibula using Sofield's technique - A case report. J Clin Orthop Trauma 2012;3(02):115-118. Doi: 10.1016/j.jcot.2012.09.003

2 Galasso O, Mariconda M, Milano C. An enlarging distal tibia osteochondroma in the adult patient. J Am Podiatr Med Assoc 2009;99(02):157-161
3 Matsumoto K, Sumi H, Shimizu K. Tibial osteochondroma causing foot pain mimicking tarsal tunnel syndrome: a case report. J Foot Ankle Surg 2005;44(02):159-162. Doi: 10.1053/j. jfas.2005.01.007

4 Chin KR, Kharrazi FD, Miller BS, Mankin HJ, Gebhardt MC. Osteochondromas of the distal aspect of the tibia or fibula. Natural history and treatment. J Bone Joint Surg Am 2000;82 (09):1269-1278

5 Spatz DK, Guille JT, Kumar SJ. Distal tibiofibular diastasis secondary to osteochondroma in a child. Clin Orthop Relat Res 1997;(345):195-197

6 Kose O, Ertas A, Celiktas M, Kisin B. Fracture of an osteochondroma treated successfully with total excision: two case reports. Cases J 2009;2:8062. Doi: 10.4076/1757-1626-2-8062

7 Bernard SA, Murphey MD, Flemming DJ, Kransdorf MJ. Improved differentiation of benign osteochondromas from secondary chondrosarcomas with standardized measurement of cartilage cap at CT and MR imaging. Radiology 2010;255(03):857-865. Doi: 10.1148/radiol.10082120

8 Gupte CM, Dasgupta R, Beverly MC. The transfibular approach for distal tibial osteochondroma: an alternative technique for excision. J Foot Ankle Surg 2003;42(02):95-98. Doi: 10.1053/ jfas.2003.50010

9 Appy-Fedida B, Krief E, Deroussen F, et al. Mitigating Risk of Ankle Valgus From Ankle Osteochondroma Resection Using a Transfibular Approach: A Retrospective Study With Six Years of Follow-Up. J Foot Ankle Surg 2017;56(03):564-567. Doi: 10.1053/ j.jfas.2017.01.029

10 Kitsoulis P, Galani V, Stefanaki K, et al. Osteochondromas: review of the clinical, radiological and pathological features. In Vivo 2008;22(05):633-646

11 Wani IH, Sharma S, Malik FH, Singh M, Shiekh I, Salaria AQ. Distal tibial interosseous osteochondroma with impending fracture of fibula - a case report and review of literature. Cases J 2009;2(01): 115. Doi: 10.1186/1757-1626-2-115

12 Ciampolini J, Gargan MF, Newman JH. Arthrodesis of the distal tibiofibular joint for a large osteochondroma in an adult. Foot Ankle Int 1999;20(10):657-658. Doi: 10.1177/107110079902001008

13 Dekker AP, Grimer RJ. Transformation of solitary osteochondroma to dedifferentiated chondrosarcoma arising in the distal radius: a case report. Musculoskelet Surg 2013;97(01):89-92. Doi: 10.1007/ s12306-012-0226-z

14 Garrison RC, Unni KK, McLeod RA, Pritchard DJ, Dahlin DC. Chondrosarcoma arising in osteochondroma. Cancer 1982;49 (09):1890-1897

15 Kim YC, Ahn JH, Lee JW. Osteochondroma of the distal tibia complicated by a tibialis posterior tendon tear. J Foot Ankle Surg 2012;51(05):660-663. Doi: 10.1053/j.jfas.2012.05.010

16 Genç B, Solak A, Kalaycıoğlu S, Şahin N. Distal tibial osteochondroma causing fibular deformity and deep peroneal nerve entrapment neuropathy: a case report. Acta Orthop Traumatol Turc 2014;48 (04):463-466. Doi: 10.3944/AOTT.2014.2741

17 Minami S, Nakatani N, Miyazaki N, Watanuki A, Honda T, Yoshida M. Spontaneous regression of a solitary osteochondroma of the distal tibia. J Orthop Sci 2009;14(05):669-671. Doi: 10.1007/ s00776-009-1362-7 Erratum published on February 27, 2019. DOI of the Erratum is 10.1055/s-0039-1683838. 